\title{
Therapeutic potential of fibroblast growth factor-2 for hypertrophic scars: upregulation of MMP-1 and HGF expression
}

\author{
Hitomi Eto ${ }^{1, *}$, Hirotaka Suga ${ }^{1, *}$, Noriyuki Aoi ${ }^{1}$, Harunosuke Kato ${ }^{1}$, Kentaro Doi ${ }^{1}$, Shinichiro Kuno ${ }^{1}$, \\ Yasuhiko Tabata ${ }^{2}$ and Kotaro Yoshimura ${ }^{1}$
}

\begin{abstract}
Although hypertrophic scars (HTSs) and keloids are challenging problems, their pathogenesis is not well understood, making therapy difficult. We showed that matrix metalloproteinase (MMP)-1 expression was downregulated in HTS compared with normal skin from the same patients, whereas type 1 and 3 collagen and transforming growth factor- $\beta$ (TGF- $\beta$ ) were upregulated. These differences, however, were not seen in cultured fibroblasts, suggesting the involvement of microenvironmental factors in the pathogenesis of HTS. Fibroblast growth factor-2 (FGF-2) highly upregulated the expression of MMP-1 and hepatocyte growth factor (HGF) in both HTS-derived and control fibroblasts; the upregulation was reversed by extracellular signal-regulated kinase (ERK) and c-Jun N-terminal kinase (JNK) inhibitors. An animal study using human HTS tissue implanted into nude mice indicated that controlled-release FGF-2 resulted in significantly less weight and decreased hydroxyproline content in HTS. Degradation of collagen fibers in FGF-2-treated HTS was also confirmed histologically. Western blotting showed that FGF-2-treated HTS expressed significantly higher MMP-1 protein than control. Decreased MMP-1 expression may be an important transcriptional change in HTS, and its reversal as well as upregulation of HGF by FGF-2 could be a new therapeutic approach for HTS.

Laboratory Investigation (2012) 92, 214-223; doi:10.1038/labinvest.2011.127; published online 26 September 2011
\end{abstract}

KEYWORDS: fibroblast growth factor-2; hypertrophic scar; matrix metalloproteinase-1

Hypertrophic scars (HTSs) are a challenging problem. Burns, traumatic injuries, and surgical procedures produce exuberant scarring that is not only aesthetically displeasing, but can also be functionally disabling, causing patients both physical and psychological distress. ${ }^{1,2}$ HTSs are linear or widespread in shape and generally red, elevated, and sometimes pruritic. ${ }^{3}$ The presence of nodules in HTS is caused by a high density of cells and collagen. Besides collagen, the major components of HTS are myofibroblasts, which are differentiated fibroblasts found in granulation tissue. A large proportion of myofibroblasts expresses smooth muscle proteins such as $\alpha$-smooth muscle actin ( $\alpha$-SMA). ${ }^{4}$

HTS can be distinguished from keloids, the other major form of excessive scarring seen in humans. There is stronger evidence for genetic predisposition in keloid formation than in HTS. ${ }^{5-7}$ Keloids are characterized by overgrowth of fibrosis beyond the boundaries of the original injury, whereas HTS remains within the boundaries of the original wound and increases in size by pushing out the edge of the scar. ${ }^{8}$ Keloids and HTS can also be distinguished by established histopathological criteria, including differences in collagen density and orientation, vascularity, and other factors. ${ }^{9} \alpha$-SMAexpressing myofibroblasts are generally absent in keloids. ${ }^{4}$ Apart from many differences, both HTS and keloid lesions likely represent aberrations in the fundamental processes of wound healing, causing excessive collagen production by fibroblast activity. ${ }^{8,10}$ Most current research on HTS and keloids involves the evaluation of protein factors that control a complex series of wound healing processes in a highly systematic manner.

There are numerous therapeutic methods for HTS or keloids that have been described, including compression therapy, gel sheeting, corticosteroid injections, surgical excision, radiation therapy, laser therapy, cryotherapy, or

\footnotetext{
${ }^{1}$ Department of Plastic Surgery, University of Tokyo School of Medicine, Tokyo, Japan and ${ }^{2}$ Department of Biomaterials, Institute for Frontier Medical Sciences, Kyoto University, Kyoto, Japan

Correspondence: Dr K Yoshimura, MD, Department of Plastic Surgery, University of Tokyo School of Medicine, 7-3-1, Hongo, Bunkyo-Ku, Tokyo 113-8655, Japan. E-mail: kotaro-yoshimura@umin.ac.jp

*These authors contributed equally to this work.

Received 25 March 2011; revised 9 May 2011; accepted 25 May 2011
} 
combinations of these, although there is no single definitive treatment modality. ${ }^{11}$ One of the most common treatments is intralesional injection of triamcinolone, which was shown to increase the production of fibroblast growth factor-2 (FGF-2; basic fibroblast growth factor) and decrease the production of transforming growth factor- $\beta$ (TGF- $\beta$ ) by human dermal fibroblasts. ${ }^{12}$

We previously demonstrated that FGF-2 released at the time of adipose injury promotes hepatocyte growth factor (HGF) secretion from adipose-derived stromal cells and/or fibroblasts and inhibits post-injury fibrogenesis. ${ }^{13}$ HGF has anti-fibrogenic effects in various organs, including heart, ${ }^{14}$ liver, ${ }^{15}$ and kidney ${ }^{16}$ and antagonizes the profibrogenetic action of TGF- $\beta$ by intercepting Smad signal transduction through diverse mechanisms. ${ }^{17}$

There are also some reports on the anti-scarring effects of FGF-2 ${ }^{18-20}$ including clinical use. Post-operative administration of FGF-2 inhibits hypertrophy and widening of scars without any serious side effects. ${ }^{21}$ These results suggested that FGF-2 treatment during wound healing may contribute to prevention of HTS and keloid formation.

Despite the possible preventive effect of FGF-2, its therapeutic potential for HTS and keloid formation has not been evaluated. We investigated whether FGF-2 has therapeutic effects on long-standing HTS. For this purpose, we comparatively evaluated the in vitro effects of FGF-2 on fibroblasts isolated from human HTS and normal skin as well as therapeutic effects on nude mouse models in which human HTS was subcutaneously implanted.

\section{MATERIALS AND METHODS}

\section{Patients and Experimental Protocol}

Nine patients (six females and three males, age 17-67 years) underwent surgical resection of HTS (Table 1). Normal skin adjacent to the scar excised during surgery was used as the control. All human and animal studies have been approved by the institutional review board, and informed consent was obtained from each patient.

Table 1 Clinical data of patients

\begin{tabular}{lcclll}
\hline Patient & Age (years) & Sex & Site & \multirow{2}{*}{$\alpha$-SMA } \\
\cline { 1 - 2 } 1 & 53 & F & Abdomen & \\
2 & 22 & TGF- $\beta$ \\
3 & 18 & F & Neck & \\
4 & 25 & M & Arm & HGF \\
5 & 62 & M & Ear & \\
6 & 17 & F & Chest & \\
7 & 36 & F & Abdomen & MMP-1 \\
8 & 67 & F & Abdomen & \\
9 & 60 & F & Abdomen & GAPDH
\end{tabular}

\section{Cell Culture}

Fibroblasts were obtained from explant cultures of normal skin and scar samples. Primary cells were cultured for 7-10 days and were defined as 'Passage 0'. Cells were passaged every week by trypsinization. Cells at passages 1-3 were used in the experiments. The culture medium was Dulbecco's modified Eagle's medium containing 10\% fetal bovine serum. The medium was replaced every 3 days. For gene expression analysis by real-time PCR, cells were cultured with or without FGF-2 $(10 \mathrm{ng} / \mathrm{ml})$ for 5 days. For the signal inhibition assay, scar-derived fibroblasts were cultured in the culture medium containing each inhibitor (ERK inhibitor, U0126; p38 protein inhibitor, SB202190; JNK inhibitor, SP600125; all from Calbiochem, La Jolla, CA, USA) at $10 \mu \mathrm{M}$ for 6 or $24 \mathrm{~h}$. Human recombinant FGF-2 was kindly supplied by Kaken Pharmaceutical (Tokyo, Japan).

\section{Quantitative Real-Time RT-PCR}

Total RNA was isolated from the samples using an RNeasy Mini Kit (Qiagen, Hilden, Germany) followed by reverse transcription. Real-time PCR was performed with the ABI 7700 sequence detection system and a SYBR Green PCR Master Mix (both from Applied Biosystems, Foster City, CA, USA). The primer sequences are shown in Table 2. Expression levels were calculated by the comparative $C_{\mathrm{T}}$ method with glyceraldehyde-3-phosphate dehydrogenase (GAPDH) as an endogenous reference gene.

\section{Scar Transplantation Model}

HTS tissue was cut into small discoidal fragments $(5 \mathrm{~mm}$ in diameter and $3 \mathrm{~mm}$ thickness) after the overlying skin

Table 2 Primer sequences used for real-time PCR

Gene Primer sequence $\left(5^{\prime}-3^{\prime}\right)$

Type 1 collagen

Forward: AGGGACACAGAGGTTCAGTGGTT Reverse: GCAGCACCAGTAGCACCATCATTा

Type 3 collagen

Forward: TATCGAACACGCAAGGCTGTGAGA Reverse: GGCCAACGTCCACACCAAATTCTT Forward: TGACAATGGCTCTGGGCTCTGTAA Reverse: TTCGTCACCCACGTAGCTGTCTT Forward: ACAATTCCTGGCGATACCTCAGCA Reverse: CGCTAAGGCGAAAGCCCTCAATTा Forward: ATGTCAGCCCTGGAGTTCCATGAT Reverse: AGCGTACCTCTGGATTGCTTGTGA Forward: AGTGACTGGGAAACCAGATGCTGA Reverse: TCAGTGAGGACAAACTGAGCCACA Forward: CATGTTCGTCATGGGTGTGAACCA Reverse: AGTGATGGCATGGACTGTGGTCAT 
and the subcutaneous fat were removed. A 7-mm centerline incision was made on the back of 6-week-old male nude mice. A discoidal HTS was then transplanted subcutaneously on the lateral side of the back. Two weeks after transplanta- tion, $10 \mu \mathrm{g}$ FGF-2 or vehicle incorporated into controlledrelease gelatin microspheres ${ }^{22,23}$ was injected into the graft. Four weeks after administration of FGF-2 or vehicle, the grafts were harvested and stained with hematoxylin and
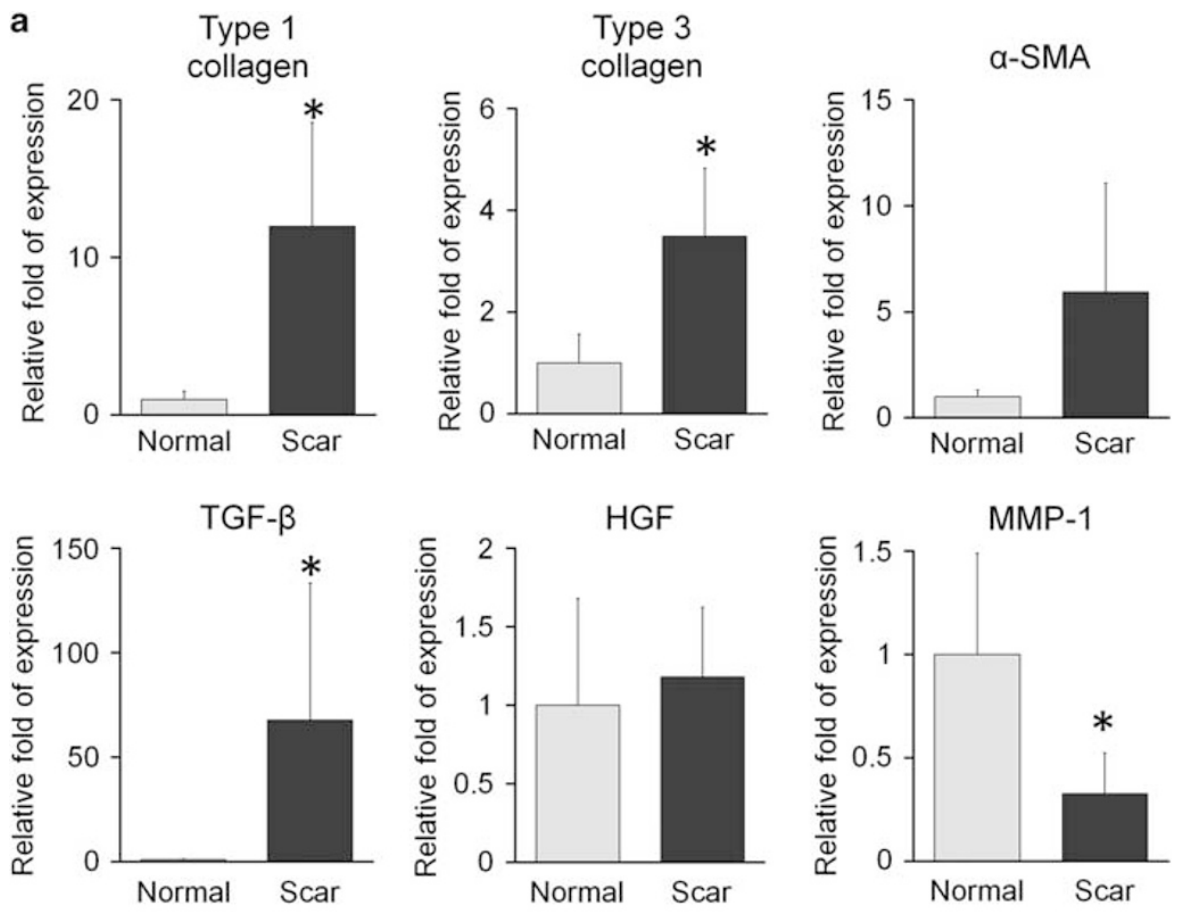

b

Type 1

collagen

Type 3

collagen
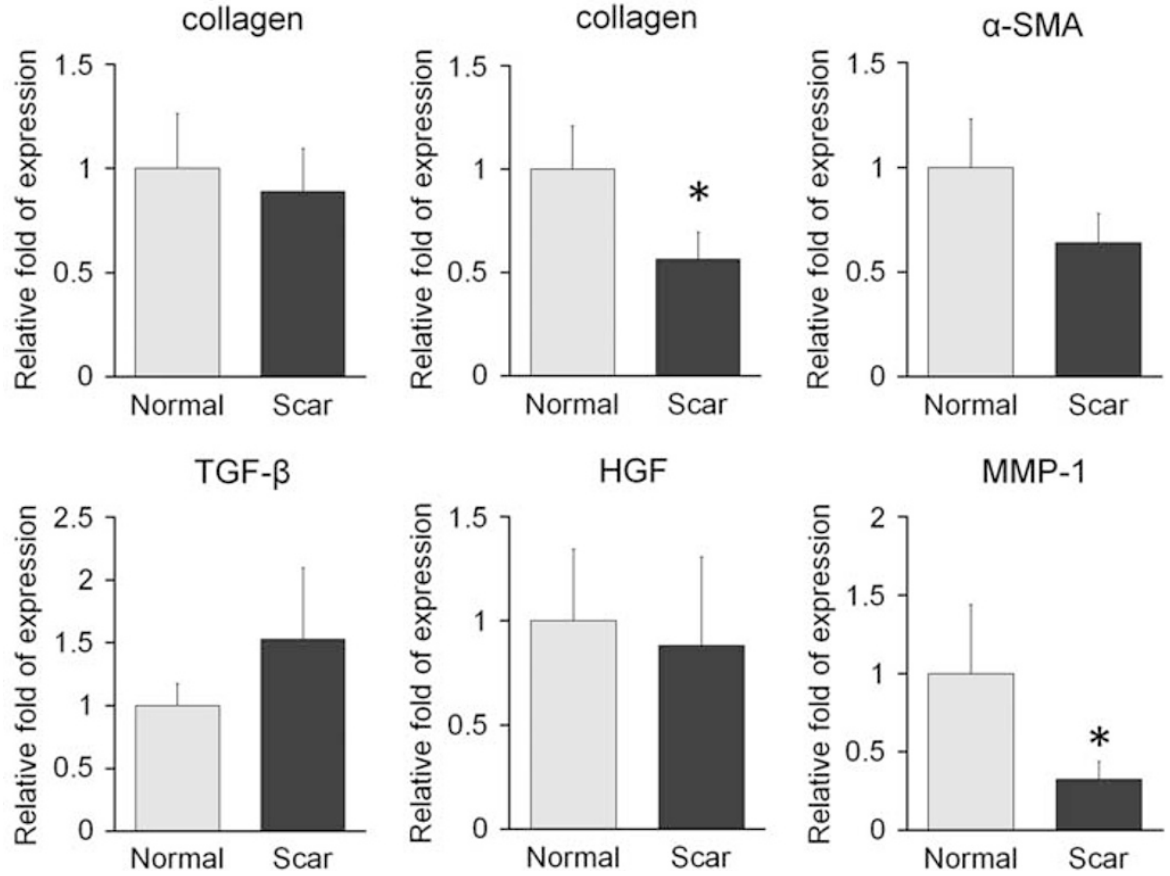

Figure 1 Gene expression in fresh tissues or cultured fibroblasts from normal skin and hypertrophic scar (HTS) tissue. (a) Gene expression in fresh tissues. Type 1 and 3 collagen and TGF- $\beta$ were highly upregulated, and MMP- 1 was downregulated in HTS. Values are expressed as mean values \pm s.e.m. $(n=5) ;{ }^{\star} P<0.05$. (b) Gene expression in cultured fibroblasts. In contrast to fresh tissues, no significant differences were observed between cultured fibroblasts derived from normal skin and HTS, except type 3 collagen and MMP-1. Values are expressed as mean values \pm s.e.m. $\left(n=7\right.$ ); ${ }^{\star} P<0.05$. 
eosin, Azan staining, and other biochemical evaluations described below. The margin of embedded tissue was easily detectable in all samples at the time of harvesting.

\section{Quantification of Hydroxyproline}

Collagen content was analyzed by measuring hydroxyproline content as described previously. ${ }^{24}$ Briefly, the samples were homogenized in $5 \mathrm{ml}$ of $6 \mathrm{~N} \mathrm{HCl}$ and hydrolyzed at $110^{\circ} \mathrm{C}$ for $24 \mathrm{~h}$. In all, $500 \mu \mathrm{l}$ of $10 \%$ alanine solution and $1 \mathrm{ml}$ of potassium borate buffer were added to the hydrolysate, which was oxidized with $1 \mathrm{ml}$ of $0.2 \mathrm{M}$ chloramine $\mathrm{T}$ solution for $25 \mathrm{~min}$. The oxidation was stopped with $3 \mathrm{ml}$ of $3.6 \mathrm{M}$ sodium thiosulfate, and $5 \mathrm{ml}$ of toluene was added to the sample, which was centrifuged. The toluene layer was removed and boiled for $30 \mathrm{~min}$. Toluene was added to the materials, and the solution was centrifuged. The toluene extract was incubated with $1 \mathrm{ml}$ of Ehrlich's reagent for $30 \mathrm{~min}$, and the absorbance was read at $560 \mathrm{~nm}$. The amount of hydroxyproline in the sample was calculated by comparison with a standard hydroxyproline curve.

\section{Western Blotting}

After the samples were homogenized in $500 \mu \mathrm{l}$ of RIPA lysis buffer (Santa Cruz Biotechnology, Santa Cruz, CA, USA), the

a

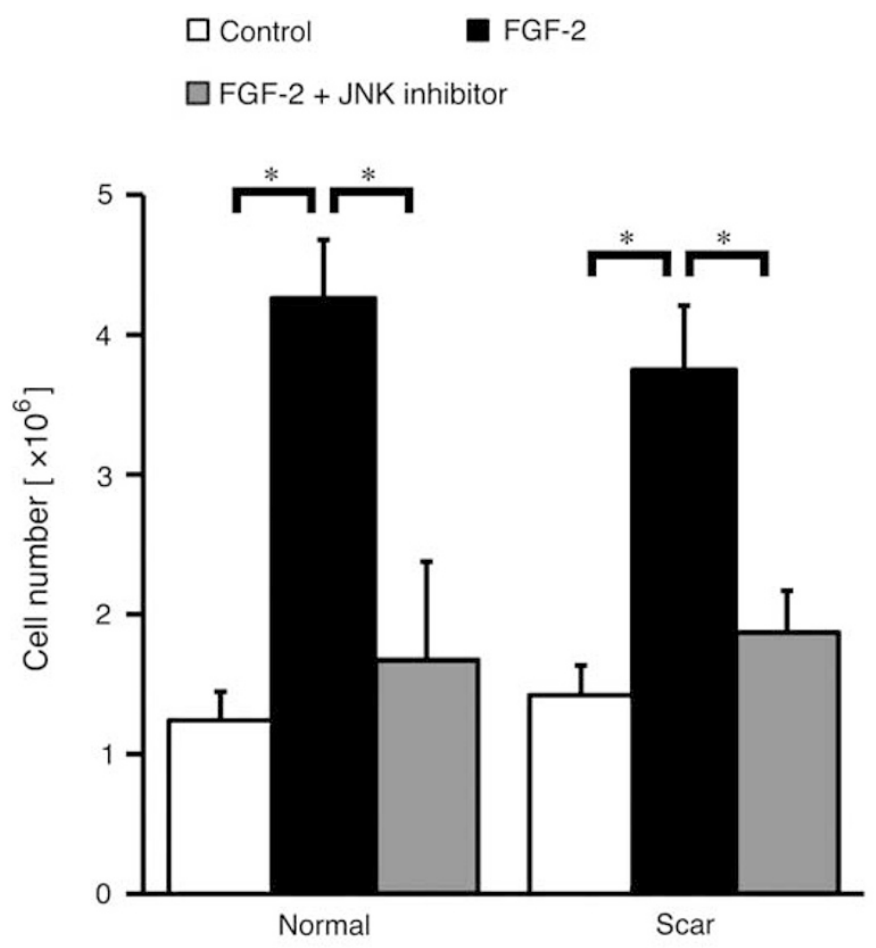

b

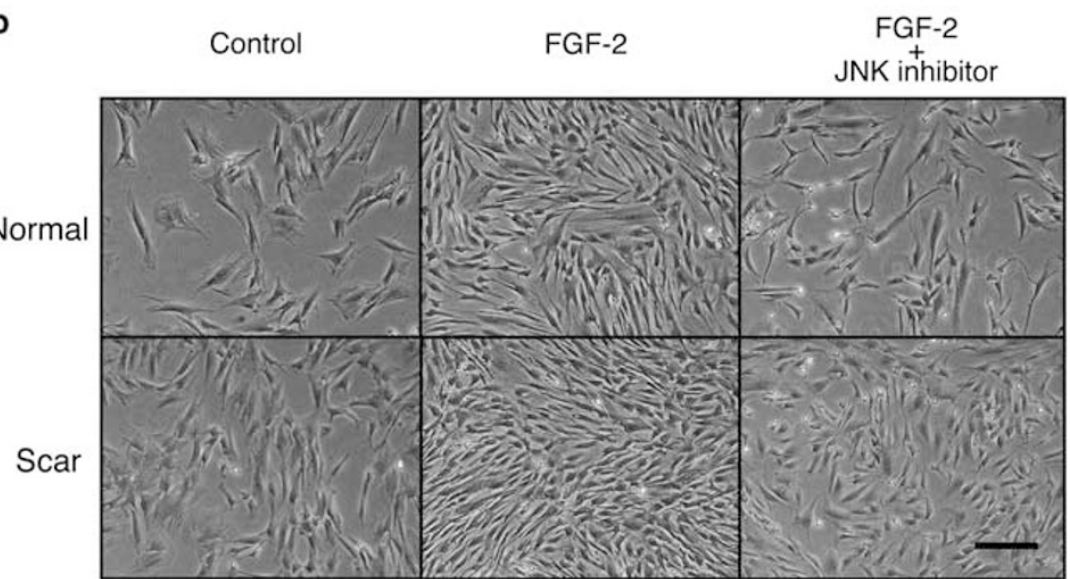

Figure 2 Effects of FGF-2 on proliferation and morphology of fibroblasts derived from normal skin or hypertrophic scars (HTSs). (a) Proliferation was promoted by FGF-2 in both normal skin-derived and HTS-derived fibroblasts and the proliferative effect of FGF-2 was inhibited by a JNK inhibitor in both cells. Values are expressed as mean values \pm s.e.m. $(n=6)$; ${ }^{\star} P<0.05$ for comparison of cell number with or without FGF-2 or JNK inhibitor.

(b) Normal skin-derived fibroblasts and HTS-derived fibroblasts were morphologically similar. Scale bar $=200 \mu \mathrm{m}$. 
aqueous layer obtained from centrifugation was collected. Protein concentrations in the samples were determined with a BCA protein assay kit (Pierce, Rockford, IL, USA), and equal amounts of protein $(10 \mu \mathrm{g})$ were loaded in each lane of an SDS-PAGE gel. The resolved proteins were transferred to a polyvinylidenedifluoride membrane
(Bio-Rad Laboratories, Hercules, CA, USA), and immunoblotting was performed with goat anti-matrix metalloproteinase-1 (anti-MMP-1) antibody (R\&D Systems, Minneapolis, MN, USA) and goat anti-GAPDH antibody (Santa Cruz Biotechnology). The GAPDH signal served as an internal control.
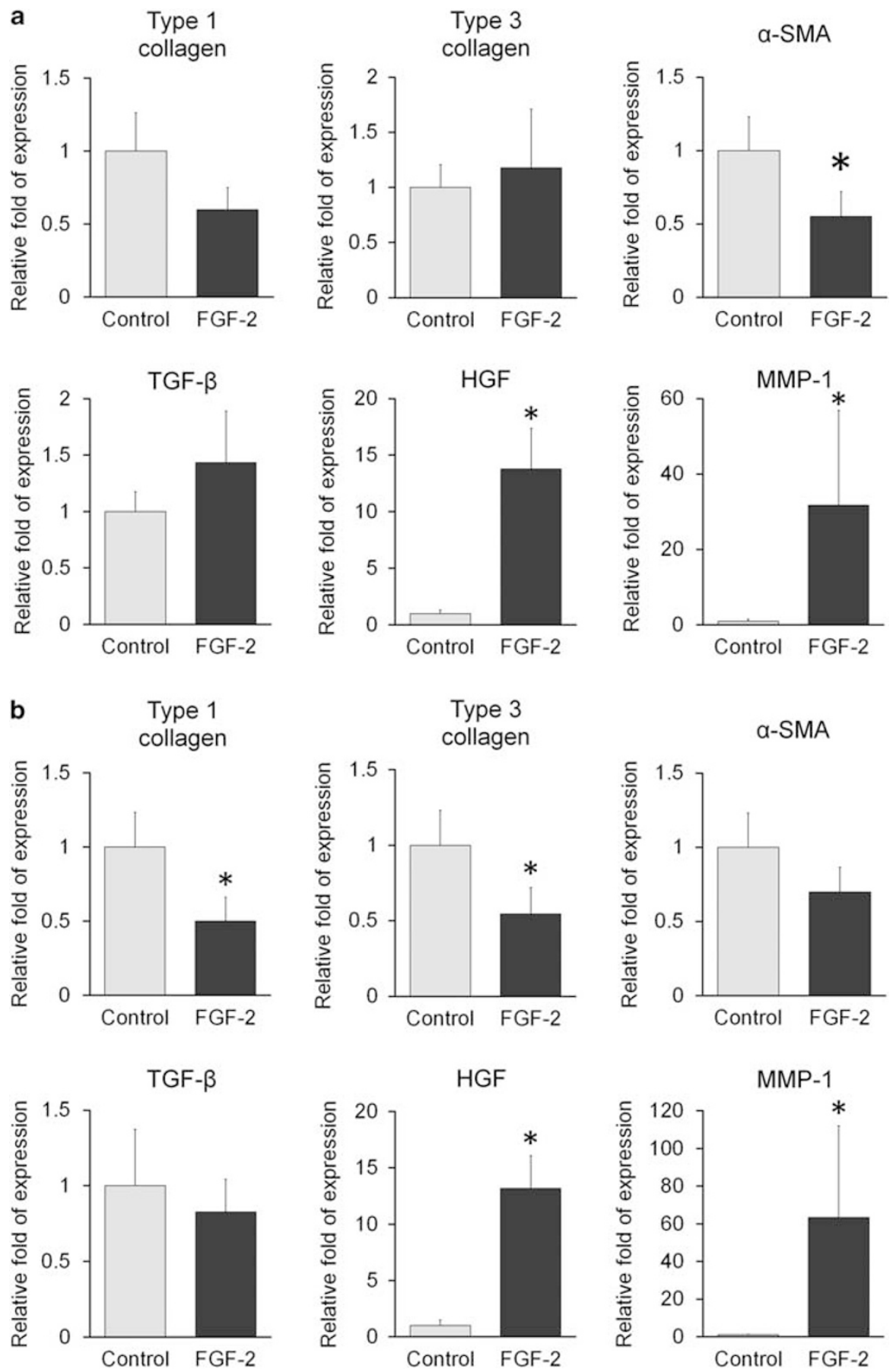

Figure 3 Effects of FGF-2 on mRNA expression of fibroblasts derived from normal skin or hypertrophic scars (HTSs). FGF-2 effects on normal skin-derived cultured fibroblasts (a) and those on HTS-derived cultured fibroblasts (b). FGF-2 downregulated expression of type 1 and 3 collagen in HTS-derived fibroblasts and upregulated expression of HGF and MMP-1 in both HTS-derived and normal skin-derived fibroblasts. Values are expressed as mean values \pm s.e.m. $(n=7) ;{ }^{\star} P<0.05$ for comparison of mRNA expression between FGF-2-treated cells and untreated control cells. 


\section{Statistical Analysis}

Results were expressed as the mean values \pm standard error of means (s.e.m.). Comparisons between two groups were performed with the Wilcoxon signed rank test. Comparisons of more than two groups were done by ANOVA with Bonferroni correction. A value of $P<0.05$ was considered significant.

\section{RESULTS}

\section{Gene Expression in HTS and Normal Skin}

Analysis of gene expression in fresh tissues indicated that type 1 and 3 collagen and TGF- $\beta$ were highly upregulated in HTS compared with normal skin. Average expression of $\alpha$-SMA and HGF was higher in HTS, although the differences were not statistically significant. In contrast, MMP-1 mRNA expression was downregulated in HTS to less than half the levels in normal skin (Figure 1a). Interestingly, however, these differences in gene expression were not seen in cultured fibroblasts between HTS and normal skin, except type 3 collagen and MMP-1, which were downregulated in HTSderived fibroblasts (Figure 1b).

\section{FGF-2 Effects on HTS-Derived Fibroblasts and Related Signaling Pathways}

FGF-2 strongly promoted cell proliferation of both HTSderived and normal skin-derived fibroblasts. The proliferation promoting effect of FGF-2 was clearly inhibited by a JNK inhibitor (Figure 2a). Morphologically, fibroblasts cultured with FGF-2 were smaller in size compared with those cultured in control medium or the JNK inhibitor, though there were no differences between HTS and normal skin (Figure 2b).

Real-time PCR indicated that FGF-2 downregulated the expression of type 1 and 3 collagen in HTS-derived fibroblasts (Figure 3). FGF-2 also downregulated $\alpha$-SMA mRNA expression in normal skin-derived fibroblasts. Most interestingly, FGF-2 highly upregulated gene expression of HGF and MMP-1 in both HTS-derived and normal skin-derived fibroblasts.

To further analyze the signaling pathways related to the upregulation of HGF and MMP-1 mRNA expression in HTSderived fibroblasts, a signal inhibition assay was performed with inhibitors of each of the mitogen-activated protein kinase (MAPK) pathways (ERK, p38, and JNK inhibitors). The administration of ERK inhibitor for both 6 and $24 \mathrm{~h}$ inhibited the upregulation of both HGF and MMP-1, and JNK inhibitors inhibited the upregulation of both HGF and MMP-1 at $24 \mathrm{~h}$, whereas the p38 inhibitor had no effect on mRNA expression of either HGF or MMP-1 in HTS-derived fibroblasts (Figure 4).

\section{Therapeutic Effects of FGF-2 Treatment on HTS in Animal Models}

Human HTS samples were subcutaneously transplanted into the back of nude mice and harvested 4 weeks after FGF-2 treatment (Figure 5a). The weight of the human HTS
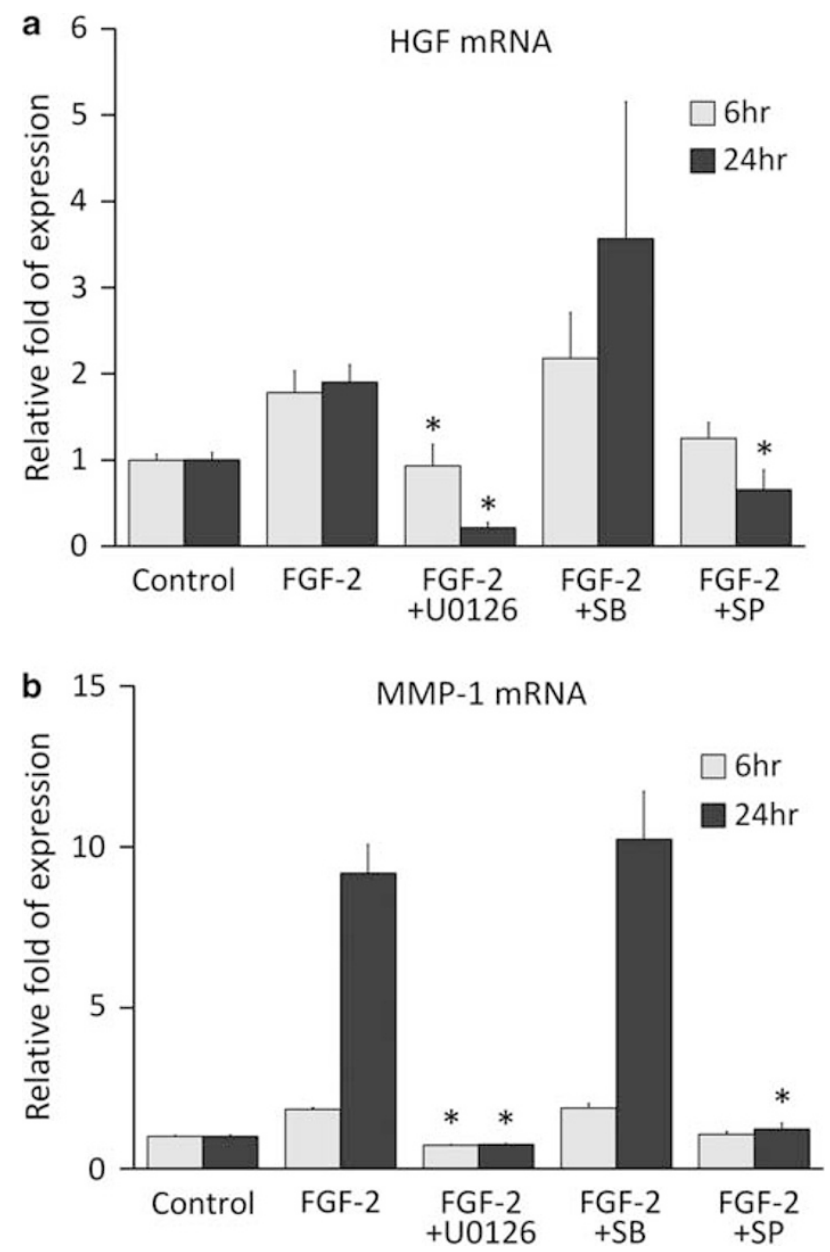

Figure 4 Selective inhibition of FGF-2 intracellular signaling pathways. (a) HGF mRNA expression. FGF-2 intracellular signaling pathways were inhibited by selective inhibitors: an ERK inhibitor, U0126; a p38 protein inhibitor, SB202190 (SB); and a JNK inhibitor, SP600125 (SP). FGF-2-induced upregulation of HGF mRNA was significantly inhibited by U0126 at 6 and $24 \mathrm{~h}$ and SP at $24 \mathrm{~h}$, whereas SB had no inhibitory effect. (b) MMP-1 mRNA expression. FGF-2-induced upregulation of MMP-1 mRNA was significantly inhibited by U0126 at 6 and $24 \mathrm{~h}$ and SP at $24 \mathrm{~h}$, whereas SB had no inhibitory effect. Values are expressed as mean values \pm s.e.m. $(n=3) ;{ }^{\star} P<0.05$ compared with FGF-2-treated cells (FGF-2) with or without an inhibitor (FGF-2 + U0126, SB, or SP).

samples was significantly smaller in the FGF-2-treated group than in the vehicle-treated group (Figure 5b). Histological analysis of the samples showed that transplanted HTS tissue was preserved well with dense collagen fibers and was distinguished clearly from surrounding tissues in the recipient site in the vehicle-treated group. In contrast, samples treated with FGF-2 had less dense collagen fibers, and the HTS tissue border with the surrounding tissue was unclear (Figure 5c).

Quantification of hydroxyproline indicated that collagen content was also significantly less in the FGF-2-treated group (Figure 6a). Western blotting showed that MMP-1 protein expression in HTS tissue was significantly increased by FGF-2 
treatment (Figure 6b). These changes in the FGF-2 group were also confirmed by Azan staining (data not shown), suggesting degradation of collagen fibers.

\section{DISCUSSION}

The therapeutic effects of FGF-2 on HTSs or keloids have not been demonstrated before, although some studies have suggested prophylactic efficacy on excessive fibrogenesis during wound healing. In this study, we evaluated the therapeutic potential of FGF-2 for long-standing HTS and analyzed the underlying mechanism.

Gene expression analysis of fresh dermal tissues in HTS and intact skin showed that HTS tissue had significantly higher mRNA expression of type 1 and type 3 collagen, important components of the extracellular matrix in HTS, ${ }^{25}$ and TGF- $\beta$, which is a pathogenic mediator in tissue fibrosis; ${ }^{26}$ but the expression of MMP-1, a collagenase that mainly digests interstitial collagen type 1 and 3 , was significantly lower; these results are consistent with previous reports analyzing tissue fibrosis. ${ }^{27,28}$ Gene expression of other collagenases, MMP-8 and MMP-13, were preliminary measured in fresh samples, but expression level of them were nearly at an undetectable level (data not shown).
On the other hand, gene expression patterns of HTSderived cultured fibroblasts were almost the same as those of normal-skin derived cultured fibroblasts, except for lower expression of type 3 collagen and MMP-1, although keloidderived fibroblasts were previously reported to differ from normal fibroblasts in expression of a large number of genes. ${ }^{29}$ Thus, HTS-derived cultured fibroblasts in this study lost many of the differences from normal dermal cells in gene expression (upregulation of collagen type 1 and 3, and TGF- $\beta$ ), suggesting possible involvement of environmental factors in the pathogenesis of HTS. However, the differences between the HTS whole tissue and HTS-derived cultured fibroblasts might be due to the other cell types residing in the whole tissue. In addition, slightly different responses to FGF-2 between HTS-derived fibroblasts and normal skin-derived fibroblasts may suggest involvement of genetic factors. Other factors regulating MMPs, such as TIMPS, might be involved, though they were not investigated in this study.

Previous studies have shown that HGF and TGF- $\beta$ are involved in the pathogenesis of fibrous diseases of various organs, and many challenges to elucidate their roles and

a

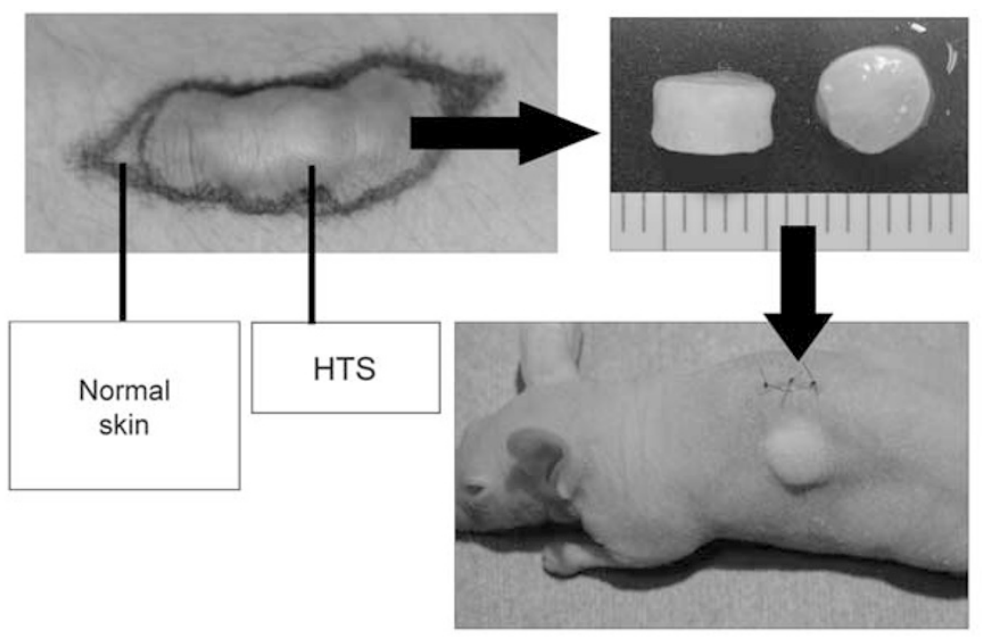

b

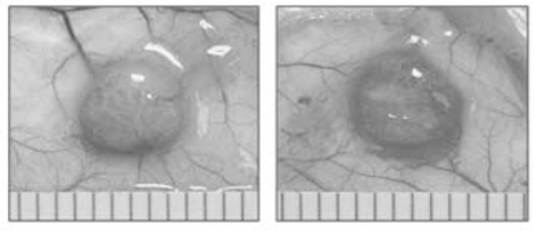

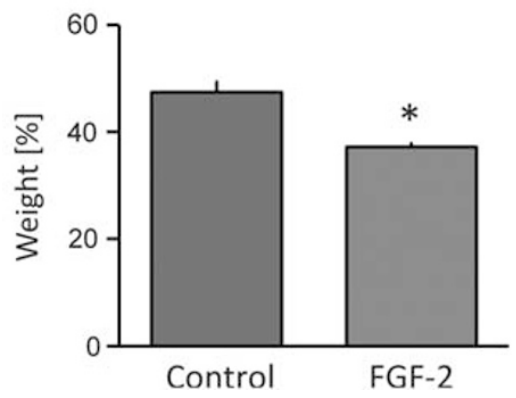

Figure 5 Therapeutic remodeling effects of FGF-2 treatment on hypertrophic scar (HTS) tissue in animal models. (a) Implantation procedure of human HTS tissue. After the epidermis and subcutaneous fat were completely removed, the HTS dermis tissues were processed to discoid shape and implanted into the subcutis in the back of mice. Adjacent intact dermis was used as control in in vitro experiments. (b) Harvested HTS specimens. Although transplanted HTS tissue was partly absorbed even without FGF-2, FGF-2 administration resulted in a more pronounced reduction of weight. Values are expressed as mean values \pm s.e.m. $(n=4) ;{ }^{*} P<0.05$. (c) Representative histology of HTS specimens with or without FGF- 2 treatment. Hematoxylin and eosin staining revealed that FGF-2-treated HTSs had less dense collagen fibers, and the border with the surrounding tissue was unclear compared with the control group. Scale bars $=500 \mu \mathrm{m}$ (top; black), $200 \mu \mathrm{m}$ (middle; yellow), and $100 \mu \mathrm{m}$ (bottom; white). 
C
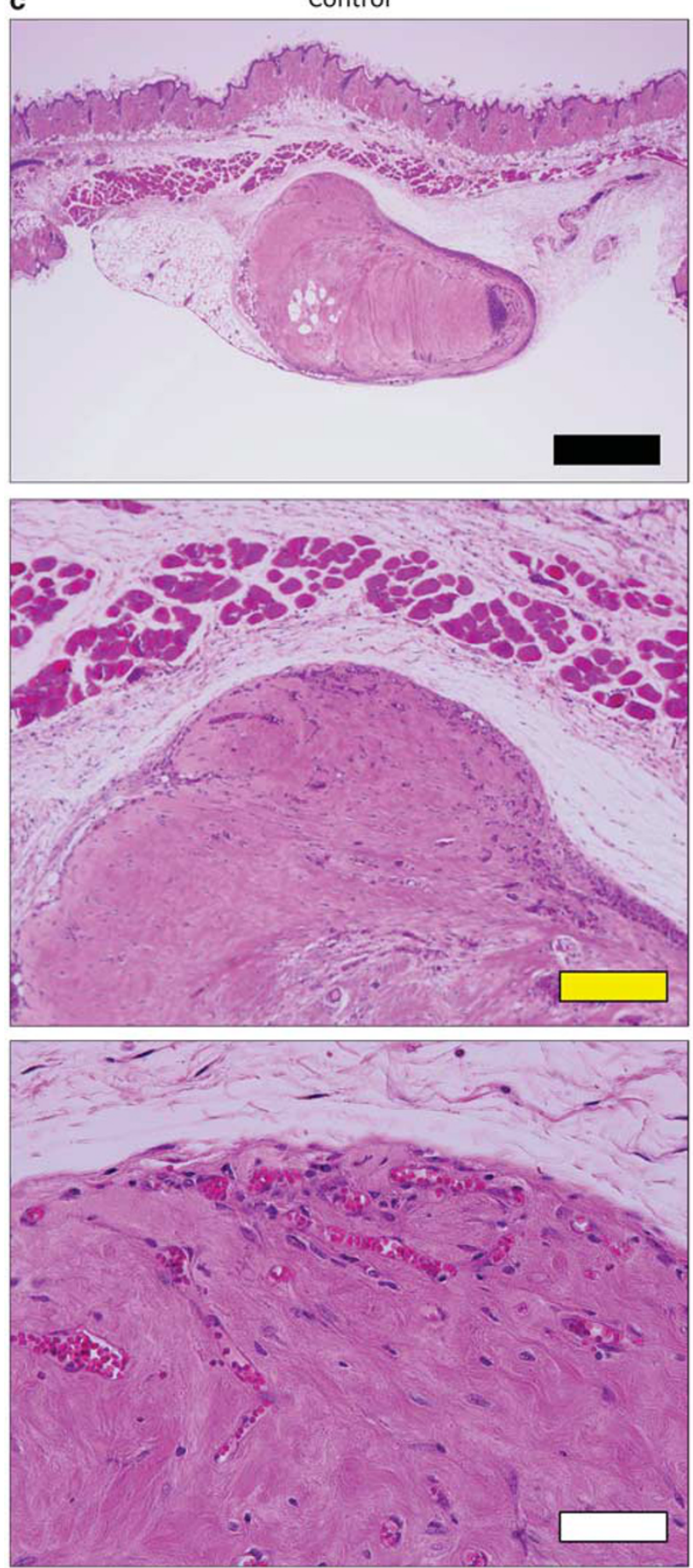
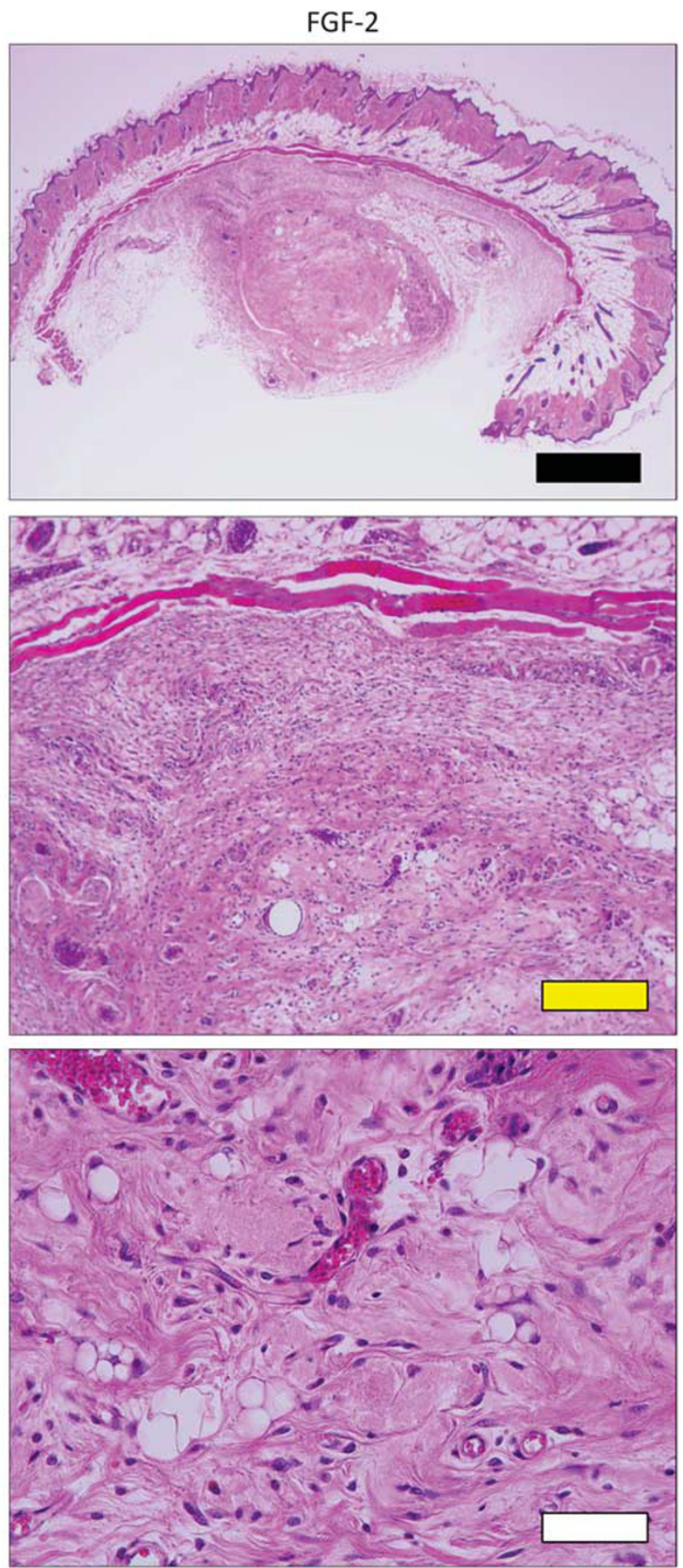

Figure 5 Continued.

relationship in fibrogenesis have been reported. ${ }^{16,17}$ TGF- $\beta 1$ is a positive regulator of fibrosis; it activates fibroblasts to become myofibroblasts and to produce large amounts of matrix proteins in various organs. ${ }^{17}$ On the other hand, HGF inhibits the TGF- $\beta 1$-mediated activation of myofibroblasts and reverses fibrogenetic processes by upregulating MMP-1. ${ }^{15,28}$ Both upregulated TGF- $\beta 1$ and decreased expression of HGF were thought to be involved in renal fibrosis. ${ }^{27}$ In this study, gene expression of HGF and MMP-1, which both have anti-fibrogenetic activity, was markedly upregulated by FGF-2 in HTS-derived and normal fibroblasts. Unlike MMP-1, upregulation of HGF mRNA expression at 6 or $24 \mathrm{~h}$ (Figure 4 ) are much lower compared to at 5 days (Figure 3), suggesting indirect effects of FGF-2 on 
a

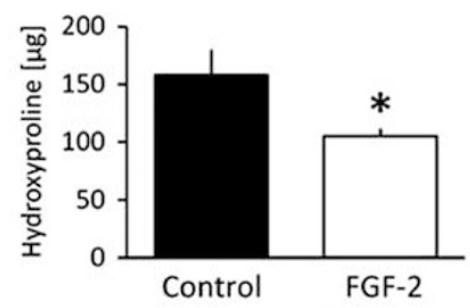

b
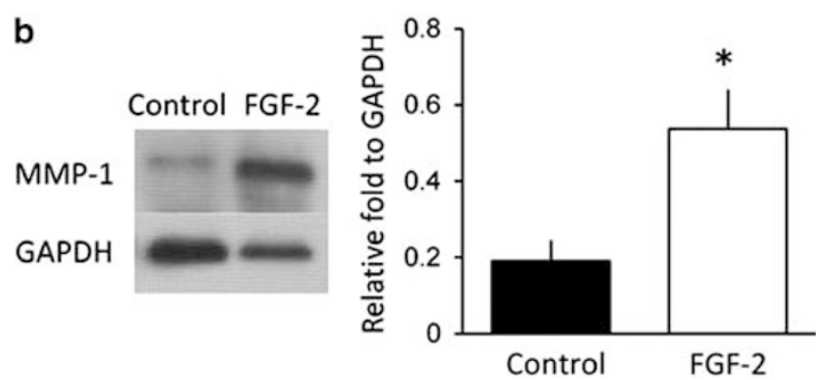

Figure 6 Biochemical evaluation of hypertrophic scar (HTS) tissue with or without FGF-2 treatment. (a) Hydroxyproline content was significantly less in FGF-2-treated HTS than in vehicle-treated HTS. Values are expressed as mean values \pm s.e.m. $(n=3)$; ${ }^{\star} P<0.05$. (b) Western blot analysis showed that MMP-1 protein in FGF-2-treated HTSs increased significantly compared with control. Values are expressed as mean values \pm s.e.m. $(n=3) ;{ }^{*} P<0.05$.

the increased upregulation of HGF at 5 days. FGF-2 was previously demonstrated to upregulate MMP-1 expression in osteoblasts ${ }^{30,31}$ and fibroblasts. ${ }^{32}$ It was also shown that upregulation of MMP-1 mRNA in osteoblasts is mediated by the MAPK pathway and completely abrogated by MAPK phosphatase (inactivates $\mathrm{ERK}=\mathrm{JNK}>\mathrm{p} 38$ MAPK), ${ }^{33}$ although the signaling mechanism in dermal fibroblasts remains to be elucidated. Using selective inhibitors of each of three signaling pathways of MAPK cascades (ERK inhibitor U0126, p38 protein inhibitor SB202190, and JNK inhibitor SP600125), we showed that FGF-2-induced upregulation of both HGF and MMP-1 mRNA expressions was abrogated by the ERK inhibitor or the JNK inhibitor in HTS-derived fibroblasts. ERK pathway seemed to be more crucial for the transcriptional activation of HGF and MMP-1, because only ERK inhibitor showed the inhibitory effect at an early stage. FGF-2 antagonizes myofibroblast activation via the ERK signaling pathway in cultured aortic valvular interstitial cells and aortic smooth muscle cells. ${ }^{34,35}$

To evaluate the therapeutic effects of FGF-2 on reducing scar tissue, we administered controlled-release recombinant FGF-2 to human HTS tissue implanted subcutaneously into the back of nude mice. Because no HTS or keloid develops in non-human animals without intervention, an ideal animal model of HTS has been unavailable, hampering relevant research. Recently, several groups attempted to observe scar formation in animal models. Yang et al ${ }^{36}$ prepared an HTS model by transplanting full-thickness human skin onto the back of nude mice, whereas Chin $e a^{37}$ stretched the murine skin with a computer-controlled device. These models were expected to reproduce scar formation process, not established HTS or keloid. In this study, we sought to evaluate a therapeutic effect on long-standing HTSs or keloids, and developed another murine model in which human HTS tissue was implanted before treatment. Previous reports also used implantation of HTS tissue, but they transplanted considerably large specimens bilaterally on the backs of nude mice, ${ }^{38,39}$ whereas we processed the HTS tissue into small discoidal pieces and implanted only one piece into the left side of the back to avoid systemic or diffusive effects of FGF-2 to the control side. The scar weight after transplantation was educed even in the vehicle-treated group as reported previously, ${ }^{38-40}$ although our reduction rate was larger perhaps because of the smaller size of the implanted tissue.

Our animal experiment results clearly indicated a therapeutic effect of FGF-2 in HTS; FGF-2 significantly decreased the scar weight and amount of collagen. Histological observation clearly indicated that collagen fibers in HTS had been degraded following FGF-2 treatment, and the mechanism likely involved increased MMP-1 mRNA and protein. FGF-2-treated tissue showed marked increase of infiltrating cells, presumably inflammatory cells; FGF-2 is primarily released in the first phase of wound healing upon injury, and thus FGF-2 injection to the tissue appears to trigger a series of subsequent wound healing process without actual wounding. ${ }^{41}$ Clinical use of FGF-2 for HTS treatment may be readily achievable, because recombinant FGF-2 protein is an approved therapeutic product (Fiblast spray ${ }^{\mathbb{R}}$, Kaken Pharmaceuticals) in Japan. It is topically used to accelerate wound healing in chronic skin ulcers. ${ }^{42}$

As a growth factor therapy, however, we must optimize the concentration and administration method. Because of the short in vivo half-life of growth factors in solution, controlled release of growth factors is needed for full effectiveness. We used gelatin hydrogel microspheres for controlled release of FGF-2 over an extended period of time as in previous studies. $^{22,23,43}$ Sequential administration of FGF-2 may be another way to achieve therapeutic effectiveness. The concentration is also important because a high dose of FGF-2 may cause an excessive inflammatory response. ${ }^{23}$ Further study is needed to optimize the parameters of FGF-2 therapy for each therapeutic purpose.

In conclusion, we provide evidence of a new therapeutic strategy, FGF-2 administration for the treatment of established HTS. Differences in gene expression between HTS and normal skin obtained from the same patients were almost diminished in cultured fibroblasts, suggesting the importance of microenvironmental factors in the pathogenesis of HTS. Pathological changes in HTS included downregulation of MMP-1 mRNA. FGF-2 increased MMP-1 and HGF expression in HTS via either ERK or JNK signaling pathway, which may be the responsible mechanism for FGF-2 effects on HTS. The efficacy of treatment using controlledrelease FGF-2 was demonstrated in animal models with implanted human HTS. 


\section{ACKNOWLEDGEMENTS}

We thank Ayako Kurata for her technical assistance. Recombinant human FGF-2 was kindly provided by Kaken Pharmaceutical (Tokyo, Japan).

\section{DISCLOSURE/CONFLICT OF INTEREST}

The authors declare no conflict of interest.

1. Bayat A, McGrouther DA, Ferguson MW. Skin scarring. BMJ 2003, 326:88-92.

2. Brown $B C$, McKenna SP, Siddhi $K$, et al. The hidden cost of skin scars: quality of life after skin scarring. J Plast Reconst Aesthet Surg 2008;61: 1049-1058.

3. Mustoe TA, Cooter RD, Gold $\mathrm{MH}$, et al. International clinical recommendations on scar management. Plast Reconstr Surg 2002;110: 560-571.

4. Kose O, Waseem A. Keloids and hypertrophic scars: are they two different sides of the same coin? Dermatol Surg 2008;34:336-346.

5. Marneros AG, Norris JE, Watanabe $\mathrm{S}$, et al. Genome scans provide evidence for keloid susceptibility loci on chromosomes $2 q 23$ and $7 p 11$. J Invest Dermatol 2004;122:1126-1132.

6. Shih B, Bayat A. Genetics of keloid scarring. Arch Dermatol Res 2010;302:319-339.

7. Nakashima M, Chung S, Takahashi A, et al. A genome-wide association study identifies four susceptibility loci for keloid in the Japanese population. Nat Genet 2010;42:768-771.

8. Butler PD, Longaker MT, Yang GP. Current progress in keloid research and treatment. J Am Coll Surg 2008;206:731-741.

9. Aarabi S, Longaker MT, Gurtner GC. Hypertrophic scar formation following burns and trauma: new approaches to treatment. PLoS Med 2007:4:1464-1470.

10. Akita $\mathrm{S}$, Akino $\mathrm{K}$, Imaizumi $\mathrm{T}$, et al. A basic fibroblast growth factor improved the quality of skin grafting in burn patients. Burns 2005; 31:855-858

11. Ogawa R. The most current algorithms for the treatment and prevention of hypertrophic scars and keloids. Plast Reconstr Surg 2010;125:557-568.

12. Carrol LA, Hanasono MM, Mikulec AA, et al. Triamcinolone stimulates bFGF production and inhibits TGF- $\beta 1$ production by human dermal fibroblasts. Dermatol Surg 2002;28:704-709.

13. Suga $\mathrm{H}$, Eto $\mathrm{H}$, Shigeura $T$, et al. IFATS collection: fibroblast growth factor-2-induced hepatocyte growth factor secretion by adiposederived stromal cells inhibits post-injury fibrogenesis through a c-Jun $\mathrm{N}$-terminal kinase-dependent mechanism. Stem Cells 2009;27:238-249.

14. Nakamura T, Mizuno S, Matsumoto K, et al. Myocardial protection from ischemia/reperfusion injury by endogenous and exogenous HGF. J Clin Invest 2000;106:1511-1519.

15. Ueki $T$, Kaneda $Y$, Tsutsui $H$, et al. Hepatocyte growth factor gene therapy of liver cirrhosis in rats. Nat Med 1999;5:226-230.

16. Liu Y. Hepatocyte growth factor in kidney fibrosis: therapeutic potential and mechanisms of action. Am J Physiol Renal Physiol 2004 287:F7-16.

17. Yang J, Dai C, Liu Y. Hepatocyte growth factor suppresses renal interstitial myofibroblast activation and intercepts Smad signal transduction. Am J Pathol 2003;163:621-632.

18. Akasaka $Y$, Ono I, Tominaga A, et al. Basic fibroblast growth factor in an artificial dermis promotes apoptosis and inhibits expression of $\alpha$-smooth muscle actin, leading to reduction of wound contraction. Wound Rep Reg 2007;15:378-389.

19. Xie JL, Bian HN, Qi SH, et al. Basic fibroblast growth factor (bFGF) alleviates the scar of the rabbit ear model in wound healing. Wound Rep Reg 2008;16:576-581.

20. Tiede S, Ernst N, Bayat A, et al. Basic fibroblast growth factor: a potential new therapeutic tool for the treatment of hypertrophic and keloid scars. Ann Anat 2008;191:33-44.

21. Ono I, Akasaka Y, Kikuchi R, et al. Basic fibroblast growth factor reduces scar formation in acute incisional wounds. Wound Rep Reg 2007;15:617-623.

22. Tabata $\mathrm{Y}$, Miyao $\mathrm{M}$, Inamoto $\mathrm{T}$, et al. De novo formation of adipose tissue by controlled release of basic fibroblast growth factor. Tissue Eng 2000;6:279-289.
23. Kimura $Y$, Ozeki $M$, Inamoto $T$, et al. Time course of de novo adipogenesis in matrigel by gelatin microspheres incorporating basic fibroblast growth factor. Tissue Eng 2002;8:603-613.

24. Kivirikko Kl, Laitinen O, Prockop DJ. Modification of a specific assay for hydroxyproline in urine. Anal Biochem 1967;19:249-255.

25. Syed $F$, Ahmadi E, lqbal SA, et al. Fibroblasts from the growing margin of keloid scars produce higher levels of collagen I and III expression compared to intra- and extra-lesional sites: clinical implications for lesional site-directed therapy. Br J Dermatol 2011;164: 83-96.

26. Pohlers D, Brenmoehl J, Löffler I, et al. TGF-beta and fibrosis in different organs - molecular pathway imprints. Biochim Biophys Acta 2009; 1792:746-756.

27. Mizuno $\mathrm{S}$, Matsumoto $\mathrm{K}$, Kurosawa $\mathrm{T}$, et al. Reciprocal balance of hepatocyte growth factor and transforming growth factor- $\beta 1$ in renal fibrosis in mice. Kidney Int 2000:57:937-948.

28. Jinnin $M$, Ihn H, Mimura $Y$, et al. Effects of hepatocyte growth factor on the expression of type I collagen and matrix metalloproteinase-1 in normal and scleroderma dermal fibroblasts. J Invest Dermatol 2005; 124:324-330.

29. Smith JC, Boone BE, Opalenik SR, et al. Gene profiling of keloid fibroblasts shows altered expression in multiple fibrosis-associated pathways. J Invest Dermatol 2008;128:1298-1310.

30. Hurley MM, Marcello K, Abreu C, et al. Transcriptional regulation of the collagenase gene by basic fibroblast growth factor in osteoblastic MC3T3-E1 cells. Biochem Biophys Res Commun 1995;214: 331-339.

31. Varghese S, Ramsby MS, Jeffrey JJ, et al. Basic fibroblast growth factor simulates expression of interstitial collagenase and inhibitors of metalloproteinases in rat bone cells. Endocrinology 1995;136: 2156-2162.

32. Xie J, Bian H, Qi S, et al. Effects of basic fibroblast growth factor on the expression of extracellular matrix and matrix metalloproteinase-1 in wound healing. Clin Exp Dermatol 2008;33:176-182.

33. Newberry EP, Willis $D$, Latifi $T$, et al. Fibroblast growth factor receptor signaling activates the human interstitial collagenase promoter via the bipartite Ets-AP1 element. Mol Endocrinol 1997;11: 1129-1144.

34. Cushing MC, Mariner PD, Liao JT, et al. Fibroblast growth factor represses Smad-mediated myofibroblast activation in aortic valvular interstitial cells. FASEB J 2008;22:1769-1777.

35. Kawai-Kowase K, Sato H, Oyama $Y$, et al. Basic fibroblast growth factor antagonizes transforming growth factor- $\beta 1$-induced smooth muscle gene expression through extracellular signal-regulated kinase $1 / 2$ signaling pathway activation. Arterioscler Thromb Vasc Biol 2004;24: 1384-1390.

36. Yang DY, Li SR, Wu JL, et al. Establishment of a hypertrophic scar model by transplanting full-thickness human skin grafts onto the backs of nude mice. Plast Reconstr Surg 2007;119:104-109.

37. Chin MS, Lancerotto L, Helm DL, et al. Analysis of neuropeptides in stretched skin. Plast Reconstr Surg 2009;124:102-113.

38. Kischer CW, Pindur J, Shetlar MR. Implants of hypertrophic scars and keloids into the nude (athymic) mouse: viability and morphology. J Trauma 1989;29:672-677.

39. Shetlar MR, Shetlar CL, Kischer CW, et al. Implants of keloid and hypertrophic scars into the athymic nude mouse: changes in the glycosaminoglycans of the implants. Connect Tissue Res 1991; 26:23-36.

40. Yang JY, Huang CY. The effect of combined steroid and calcium channel blocker injection on human hypertrophic scars in animal model: a new strategy for the treatment of hypertrophic scars. Dermatol Surg 2010;36:1942-1949.

41. Eto $H$, Suga $H$, Aoi $N$, et al. Adipose injury-associated factors activate adipose stem/stromal cells, induce neoangiogenesis, and mitigate hypoxia in ischemic tissues. Am J Pathol 2011;178: 2322-2332.

42. Ichioka S, Ohura N, Nakatsuka T. The positive experience of using a growth factor product on deep wounds with exposed bone. J Wound Care 2005;14:105-109.

43. Kimura $\mathrm{Y}$, Ozeki $\mathrm{M}$, Inamoto $\mathrm{T}$, et al. Adipose tissue engineering based on human preadipocytes combined with gelatin microspheres containing basic fibroblast growth factor. Biomaterials 2003; 24:2513-2521. 